\title{
Características demográficas del síndrome de Down en Navarra. Evolución del diagnóstico pre y postnatal durante el periodo 1991-2009
}

\section{Demographic characteristics of Down's syndrome in Navarra. Trends of pre and postnatal diagnosis for the period 1991-2009}

\author{
M.A. Ramos-Arroyo ${ }^{1}$, M. Lizarraga Rojas ${ }^{1}$, B. Hernández Charro ${ }^{1}$, \\ M.D. Martínez Jaurrieta ${ }^{1}$, J. Zabaleta Jurío ${ }^{2}$, A. Alonso Sánchez ${ }^{1}$
}

\section{RESUMEN}

Este estudio describe la evolución del diagnóstico pre y postnatal del síndrome de Down (SD) en la Comunidad Foral de Navarra desde 1991 a 2009 y analiza su impacto preventivo a nivel poblacional, así como los cambios asociadas a dicha cromosomopatía.

En ausencia de diagnóstico prenatal del SD, el cambio en la edad materna desde 1991 a 2009 hubiera conllevado un aumento del $50 \%$ en el número de nacimientos con esta cromosomopatía. Sin embargo, el incremento de la tasa de detección prenatal (15,8\% durante 19911994 y $64,3 \%$ entre $2006-2009$ ) ha condicionado una ligera tendencia descendiente de su prevalencia al nacimiento, no estadísticamente significativa, y un aumento en la edad media de sus madres $(32,75 \pm 5,02$ y $34,8 \pm 4,82$ años en el primer y último periodos, respectivamente). El porcentaje de SD nacidos vivos, hijos de madres menores de 35 años, fue del $66 \%$ en 1991-2004 y del $45 \%$ en 2006 2009. Casi una quinta parte de las gestantes rechazaron el cribado bioquímico o la amniocentesis y un $17 \%$ de los nacimientos con SD tuvieron un resultado de cribado positivo, pero las madres decidieron continuar el embarazo. Estos resultados sugieren que, a pesar de implementar nuevos y más sensibles test de cribado, la incidencia del SD puede mantenerse relativamente alta en nuestra población, circunstancia a tener en cuenta tanto en la elaboración de los planes de prevención antenatal como de los de su cuidado postnatal.

Palabras clave. Amniocentesis Diagnóstico prenatal. Cobertura. Cribado. Demografía. Síndrome de Down.

\begin{abstract}
This study describes the development of pre and postnatal diagnosis of sindrome de Down (SD) in the Autonomous Community of Navarre from 1991 to 2009 and assesses its preventive impact in the population, as well as to associated socio-demographic changes.

In the absence of a prenatal diagnosis for DS, the change in maternal age from 1991 to 2009 would have caused a $50 \%$ increase in births with this disorder. However, the antenatal rate detection of DS increased from $15.8 \%$ in $1991-4$ to $64.3 \%$ in $2006-9$, giving rise to a decreasing incidence trend, not statistically significant, during the study period and to a higher mean age of mothers of live births with DS $(32.75 \pm 5,02$ and $34.8 \pm 4,82$ years during the first and second periods of the study, respectively). The proportion of young mothers ( $<35$ years) of live births with DS was 66\% in 1991-4 and 45\% in 20069. Close to one fifth of the total population of pregnant women, however, did not want to go through a maternal screening test or amniocentesis. Seventeen per cent of all live births with DS had a positive screening test, but mothers decided to continue pregnancy. These results suggest that, despite the application of new and more sensitive prenatal screening tests, the incidence of DS may still be relatively high in our population, an important factor to be considered for future antenatal preventive programs and adequate postnatal care.
\end{abstract}

Key words. Amniocentesis. Down syndrome. Coverage. Demography. Prenatal diagnosis. Screening.
1. Servicio de Genética. Complejo Hospitalario de Navarra. Pamplona.

2. Servicio de Ginecología. Complejo Hospitalario de Navarra. Pamplona.

Recepción: 18 de noviembre de 2012

Aceptación provisional: 14 de enero de 2013

Aceptación definitiva: 26 de marzo de 2013

\author{
Correspondencia: \\ María A. Ramos-Arroyo \\ Servicio de Genética \\ Complejo Hospitalario de Navarra \\ C/ Irunlarrea, 4 \\ 31008 Pamplona \\ E-mail: mramosar@cfnavarra.es
}




\section{INTRODUCCIÓN}

El síndrome de Down (SD) es la cromosomopatía más frecuente al nacimiento y se asocia mayoritariamente a la edad avanzada de la madre'. El primer programa de prevención, establecido en los años 80 , se basada en el riesgo asociado a este factor, indicándose la realización de un proceso invasivo, mayoritariamente la amniocentesis, a gestantes de más de 35 años. Durante las dos últimas décadas se han desarrollado diversos programas de cribado prenatal de cromosomopatías con el objetivo de identificar más específicamente las gestaciones a riesgo y disminuir las complicaciones derivadas de las técnicas invasivas de diagnóstico. La utilización de diversos marcadores bioquímicos de segundo trimestre en suero materno (alfa-fetoproteína, human chorionic gonadotrophic -B-HCG-, estriol no conjugado o inhibina $A$ ), junto con la medición de la traslucencia nucal en el primer trimestre de gestación ${ }^{2,3}$, contribuyeron a incrementar el valor predictivo del cribado. Hoy en día, otros marcadores bioquímicos de primer trimestre (proteína A plasmática asociada al embarazo -PAPPA-y la fracción libre de la B-HCG) confieren una capacidad de detección del 80-90\% con un 2-3\% de falsos positivos ${ }^{4}$. La puesta en marcha de estos programas de cribado de SD ha sido un proceso progresivo a nivel internacional durante las últimas décadas, y sus resultados variables, dependientes de factores geodemográficos ${ }^{5-7}$.

En el Estado Español, el primer programa de diagnóstico prenatal de cromosomopatías con indicación de amniocentesis a madres mayores de 35 años, se inició durante los primeros años de 1980. Su desarrollo a nivel nacional fue desigual, dependiendo de la región o autonomía, al igual que lo fue la puesta en marcha de los posteriores programas de cribado bioquímico y/o ecográfico del $\mathrm{SD}^{8}$. Este estudio analiza, de forma retrospectiva, el desarrollo, cobertura y resultados del diagnóstico prenatal en la Comunidad Foral de Navarra, con el objetivo de evaluar su impacto a nivel poblacional y los cambios socio-demográficos asociados.

\section{MATERIAL Y MÉTODOS}

El estudio analiza 19 años (1991-2009) de desarrollo del programa de cribado y diagnóstico prenatal de SD del Servicio Navarro de Salud-Osasunbidea (SNS-O) en la Comunidad Foral de Navarra. Los datos correspondientes al cribado de SD, cariotipos en líquido amniótico y sangre periférica, y producto final de las gestaciones se obtuvieron a través de la historia clínica informatizada del SNS-O, de las bases de datos del servicio de Genética del Complejo Hospitalario de Navarra y del Registro de Anomalías Congénitas y Enfermedades Hereditarias de Navarra (RACEHNA). La información sobre los recién nacidos totales, interrupciones de embarazo (IVE) y sus correspondientes edades maternas se obtuvieron a través del servicio de Estadística de Navarra.

La Comunidad Foral de Navarra tiene en la actualidad una población de 650.000 habitantes y cerca de 7.000 nacimientos anuales. El SNS-O atiende a más del $90 \%$ de los embarazos y partos de la Comunidad y dispone de un sistema informático unificado de historia clínica desde el año 2002. El servicio de Genética del Complejo Hospitalario de Navarra es el único centro de estudios genéticos y coordina los programas de diagnóstico prenatal y cribado de cromosomopatías desde su inicio, así como el Registro de Anomalías Congénitas y Enfermedades Hereditarias de Navarra (RACEHNA) ${ }^{9}$. Este registro, de base poblacional, recoge información pre y postnatal de todos los recién nacidos vivos, muertos e interrupciones voluntarias del embarazo con defectos congénitos y/o enfermedades hereditarias nacidos en Navarra desde el año 1991.

Los servicios de Consejo Genético y Diagnóstico Prenatal en Navarra se iniciaron en el año 1991, tras la puesta en marcha de un Programa de Prevención de las Minusvalías, promovido por el Real Patronato de Prevención de Minusvalías. Inicialmente los estudios citogenéticos en líquido amniótico se realizaron fuera de la comunidad foral. En 1995 se establecieron las bases e infraestructura necesarias 
para el desarrollo de un proyecto específico y centralizado de diagnóstico prenatal de cromosomopatías a nivel autonómico, con dos indicaciones de amniocentesis para SD: edad materna $\geq 35$ años con fecha de última regla y resultado positivo del test de cribado bioquímico en suero materno.

El programa de cribado de SD en Navarra se inició como un proyecto piloto en el año 1994, extendiéndose, posteriormente a toda la comunidad foral. Para ello se establecieron tres nodos de extracción de muestras de sangre (Estella, Tudela y Pamplona), un laboratorio único para su procesamiento (Laboratorio de Análisis Clínicos del antiguo hospital Virgen del CaminoHVC) y un centro de coordinación global del programa (servicio de Genética del HVC). Se trataba de un test de cribado de segundo trimestre, que incluía la determinación de los niveles de alfa-feto proteína y beta-HCG en suero materno durante las semanas 15-16 de gestación y la estimación de riesgo combinado con el correspondiente a la edad materna. La cifra de corte de riesgo se estableció en 1:270. En el año 2006, el test de cribado incorporó la medición ecográfica de la traslucencia nucal fetal durante la semana 10-13 de gestación como factor adicional de estimación de riesgo, incluyéndose, además, el efecto de otros factores modificadores como la diabetes, el tipo de gestación, el peso y la etnia. El programa participa en el sistema de control de calidad externo (UKA-NEQAS) desde el año 2004. Con el fin de compartir e integrar toda esta información, se diseñó una ficha específica de cribado de cromosomopatías, incluida en la historia clínica informatizada de cada gestante. En ella se recoge la información ecográfica, bioquímica, genética y pediátrica de cada gestación. Esta ficha permite, además, programar automáticamente la fecha de realización del cribado o amniocentesis, estimar el riesgo para síndrome de Down y otras cromosomopatías, especificar las indicaciones pertinentes en cada caso, y recoger los resultados de la amniocentesis y del final de la gestación.

\section{Variables e indicadores}

Se analizaron las siguientes variables correspondientes a las gestaciones controladas durante el periodo de estudio a través del SNS-O: edad materna, riesgo estimado para SD según el resultado del test de cribado bioquímico de $2^{\circ}$ trimestre, realización de amniocentesis, cariotipo fetal, producto de la gestación [recién nacido vivo (RNV), recién nacido muerto (RNM), aborto espontáneo (AE) o interrupción voluntaria del embarazo (IVE)] y defectos al nacimiento.

Los indicadores analizados en relación al SD fueron: a) prevalencia total [ $n^{\circ}$ de casos con SD (vivos, muertos e IVE de segundo trimestre de gestación)/población total $\left(n^{\circ}\right.$ total recién nacidos e IVE de segundo trimestre de gestación], b) Prevalencia al nacimiento [ $\mathrm{n}^{\mathrm{o}}$ de recién nacidos (vivos y muertos) con $\mathrm{SD} / \mathrm{n}^{\circ}$ total recién nacidos].

\section{Análisis estadístico}

Los datos se presentan con medias; las variables categóricas se expresan en números absolutos (porcentaje). La tendencia de la prevalencia del SD se analizó con el test de regresión de Poisson. Con el fin de comparar los cambios habidos entre el inicio y el final del estudio se definió al primero como el periodo previo a la puesta en marcha del programa de DP (1991-1994) y al segundo como aquél de la misma duración que incluye los últimos años de desarrollo del mismo (2006-2009). Los datos de prevalencia por 10.000 se presentan en periodos acumulados trienales. El cálculo del número de recién nacidos con SD en función de la edad materna se realizó según Morris y $\mathrm{col}^{10}$.

\section{RESULTADOS}

\section{Datos demográficos}

Entre 1991-2009 se registraron un total de 105.589 nacimientos, con un incremento medio anual del 3\%. El 23\% de los mismos correspondió a madres con edades iguales o superiores a los 35 años, variando entre 
el $13,1 \%$ en 1991 y el $30,1 \%$ en 2009 . La edad media materna fue de $29,47 \pm 4,68$ años en 1991 y de $31,84 \pm 5,07$ años en 2009 . La figura la representa el cambio en la distri- bución de la edad materna de los niños nacidos en los primeros (1991-4) y últimos (2006-9) años del periodo de estudio.

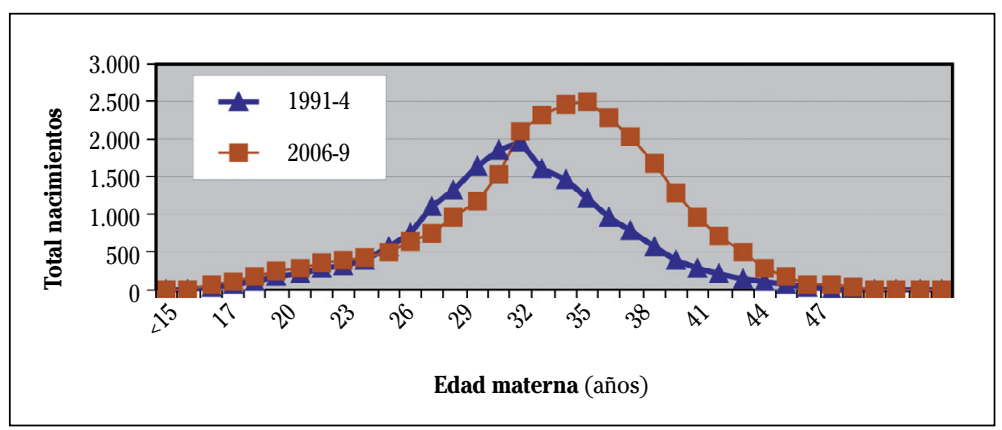

Figura 1a. Cambios en la distribución de la edad materna de todos los nacimientos en Navarra entre los periodos 1991-4 y 2006-9.

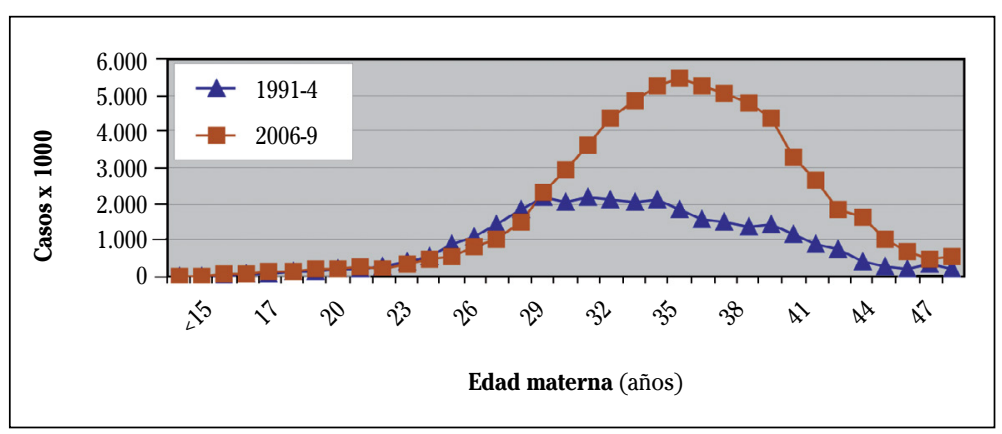

Figura 1b. Estimación del incremento del número de gestaciones con síndrome de Down (cálculo realizado según estimación de Morris y $\mathrm{col}^{10}$ ) como consecuencia de los cambios en la distribución de la edad maternal entre los periodos 1991-4 y 2006-9 en Navarra.

\section{Cobertura del programa de cribado y diagnóstico prenatal del SD}

Las solicitudes de consulta de diagnóstico prenatal, amniocentesis y test de cribado de cromosomopatías experimentaron un incremento llamativo y continuado a lo largo de todo el periodo de estudio, siendo 2,$3 ; 3,8$ y 5 veces mayor, respectivamente, en la última que en la primera parte del mismo. El porcentaje de tests de cribado y amniocentesis en relación al número total de partos fue de un $20,2 \%$ y un $5 \%$ en 1995 , y de un $59,2 \%$ y $12,1 \%$ en 2009 (Fig. 2). La sensibilidad del cribado para SD durante el periodo del estudio fue de un $70 \%$ con un $5 \%$ de falsos positivos. 


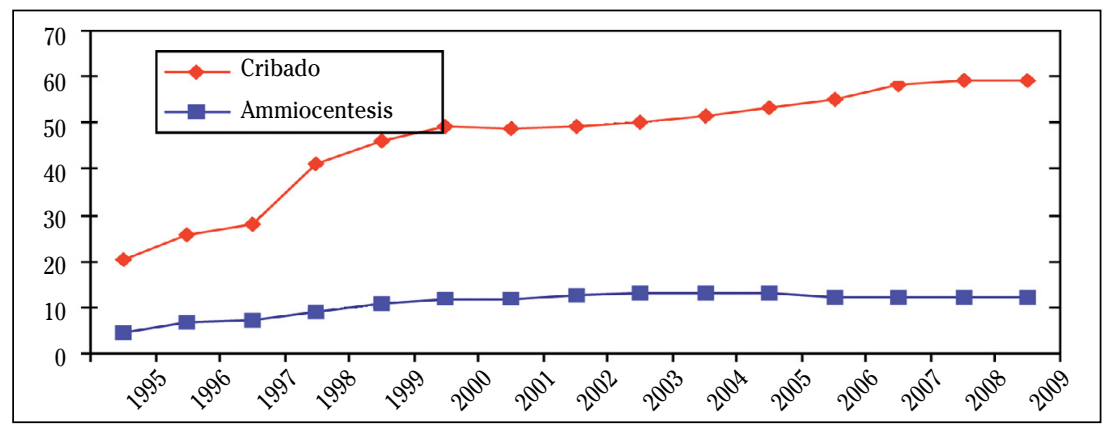

Figura 2. Cobertura del programa de diagnóstico prenatal de síndrome de Down en Navarra. Porcentaje de tests de cribado y amniocentesis por $n^{\circ}$ de partos desde 1995 a 2009.

El análisis de la decisión de las embarazadas (21.097 gestaciones consecutivas) en el momento de realizar la ecografía fetal de primer trimestre (11-13 de gestación), muestra que durante el periodo 2006-2009, un $70,8 \%$ optaron por realizar un cribado de SD, oscilando entre un $81,2 \%$ entre las menores de 35 años y un $24,1 \%$ entre las de 38 o más (Tabla 1). Casi un 55\% de las madres de este último grupo decidieron inicialmen- te realizar una amniocentesis y posterior estudio citogenético del líquido amniótico, en tanto que sólo eligieron esta opción un $31,2 \%$ de las del grupo comprendido entre los 35 y 37 años. Un 18,4\% del total de la población gestante rechazó cualquier tipo de cribado o diagnóstico de cromosomopatías, a excepción de la ecografía fetal de segundo trimestre, siendo ligeramente superior entre las madres de más edad.

Tabla 1. Opciones de prevención de síndrome del Down elegidas por las embarazadas en la semana 11-13 de gestación en Navarra por grupos de edad durante el periodo 2006-2009

\begin{tabular}{|c|c|c|c|c|}
\hline \multirow[b]{2}{*}{ Plan a seguir (opción elegida) } & \multicolumn{3}{|c|}{ Edad materna (años) } & \multirow{2}{*}{ Total } \\
\hline & $<35$ & 35-37 & $\geq 38$ & \\
\hline \multirow{2}{*}{ No desea seguir } & 2.806 & 669 & 360 & 3.865 \\
\hline & $18,0 \%$ & $18,1 \%$ & $21,0 \%$ & $18,4 \%$ \\
\hline \multirow{2}{*}{ Ammiocentesis } & 117 & 1.152 & 1.016 & 2.285 \\
\hline & $0,8 \%$ & $31,2 \%$ & $54,9 \%$ & $10,8 \%$ \\
\hline \multirow{2}{*}{ Cribado bioquímico } & 12.627 & 1.874 & 446 & 14.947 \\
\hline & $81,2 \%$ & $50,7 \%$ & $24,1 \%$ & $70,8 \%$ \\
\hline \multirow{2}{*}{ Total } & 15.550 & 3.695 & 1.852 & 21.097 \\
\hline & $100,0 \%$ & $100,0 \%$ & $100,0 \%$ & $100,0 \%$ \\
\hline
\end{tabular}

\section{Diagnóstico y prevención del SD}

Durante el periodo de estudio se realizaron un total de 9.682 amniocentesis, detectándose 427 alteraciones cromosómicas (4,4\%). Un $50 \%$ de ellas fueron trisomías del cromosoma 21 y un $25 \%$ adicional correspondieron a otras aneuploidías. Más de la mitad de estas gestaciones se interrumpieron voluntariamente y un $44 \%$ fueron recién nacidos vivos. La indicación mayoritaria de amniocentesis a lo largo de todo el periodo fue la edad materna. Durante los años 2006-
2009, esta indicación correspondió al 74\% de las pruebas invasivas, un $17 \%$ se realizaron por resultado positivo del test de cribado y un 5\% tras detectarse un defecto fetal mayor por ecografía. La tasa de detección de alteraciones cromosómicas fue significativamente inferior en el grupo de indicación exclusiva por edad materna. El 46\% del total de cromosomopatías se identificaron entre el $22 \%$ de las amniocentesis indicadas por test de cribado positivo o defectos ecográficos del desarrollo fetal (Fig. 3). 


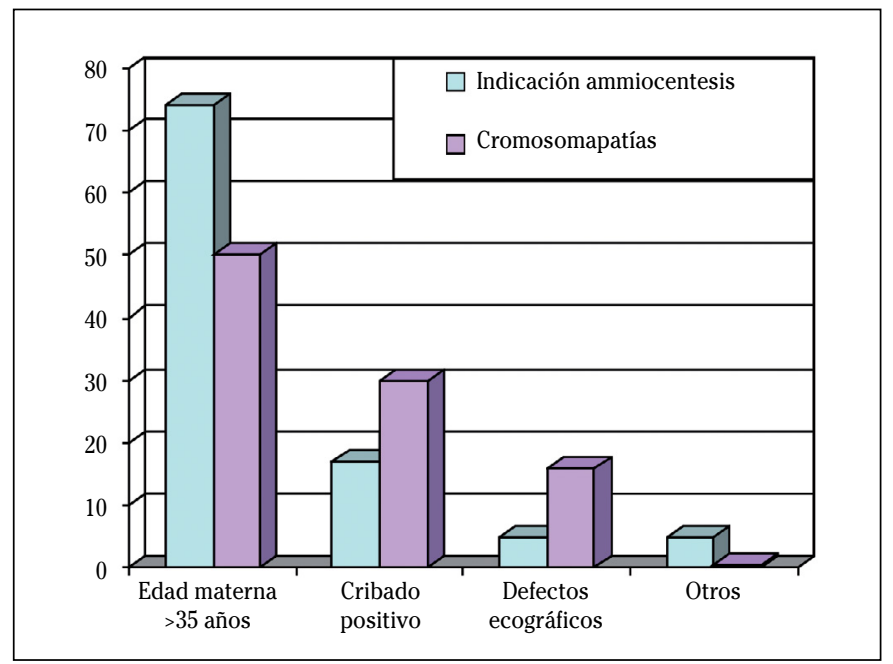

Figura 3. Porcentaje de indicaciones de amniocentesis y sus correspondientes tasas de detección de alteraciones cromosómicas en Navarra.

Tabla 2. Casos con síndrome de Down en Navarra identificados desde 1991 a 2009 por tipo de nacimiento

\begin{tabular}{ccccccccc}
\hline Año & $\begin{array}{c}\text { Total } \\
\text { nacidos }\end{array}$ & Total & $\begin{array}{c}\text { Nacidos } \\
\text { vivos }\end{array}$ & $\begin{array}{c}\text { Nacidos } \\
\text { muertos }\end{array}$ & $\begin{array}{c}\text { Interrupciones } \\
\text { del embarazo }\end{array}$ & $\begin{array}{c}\text { Abortos } \\
\text { espontáneos }\end{array}$ & $\begin{array}{c}\text { Prevalencia } \\
\text { total }\end{array}$ & $\begin{array}{c}\text { Prevalencia } \\
\text { nacimiento }\end{array}$ \\
\hline 1991 & 4.789 & 11 & 10 & 1 & 0 & 0 & 22,75 & 22,97 \\
\hline 1992 & 4.549 & 9 & 8 & 0 & 1 & 0 & 19,60 & 17,59 \\
\hline 1993 & 4.688 & 5 & 4 & 0 & 1 & 0 & 10,57 & 8,53 \\
\hline 1994 & 4.650 & 13 & 9 & 0 & 3 & 1 & 27,69 & 19,35 \\
\hline 1995 & 4.627 & 15 & 13 & 0 & 1 & 1 & 32,11 & 28,10 \\
\hline 1996 & 4.830 & 11 & 3 & 0 & 7 & 1 & 22,56 & 6,21 \\
\hline 1997 & 4.967 & 14 & 6 & 0 & 8 & 0 & 27,92 & 12,08 \\
\hline 1998 & 4.952 & 15 & 3 & 0 & 12 & 0 & 30,01 & 6,06 \\
\hline 1999 & 5.122 & 12 & 4 & 0 & 8 & 0 & 23,21 & 7,81 \\
\hline 2000 & 5.262 & 14 & 6 & 0 & 7 & 1 & 26,37 & 11,40 \\
\hline 2001 & 5.710 & 27 & 10 & 0 & 16 & 1 & 46,86 & 17,51 \\
\hline 2002 & 5.802 & 11 & 6 & 0 & 5 & 0 & 18,79 & 10,34 \\
\hline 2003 & 6.180 & 9 & 2 & 0 & 7 & 0 & 14,43 & 3,24 \\
\hline 2004 & 6.293 & 20 & 11 & 0 & 8 & 1 & 31,46 & 17,48 \\
\hline 2005 & 6.149 & 19 & 4 & 0 & 14 & 1 & 30,62 & 6,51 \\
\hline 2006 & 6.551 & 25 & 9 & 1 & 15 & 0 & 37,79 & 15,26 \\
\hline 2007 & 6.595 & 32 & 11 & 0 & 20 & 1 & 48,01 & 16,68 \\
\hline 2008 & 7.029 & 31 & 8 & 1 & 19 & 3 & 43,72 & 12,80 \\
\hline 2009 & 6.844 & 24 & 12 & 0 & 11 & 1 & 34,73 & 17,53 \\
\hline & & & & & & & &
\end{tabular}


Tabla 3. Evolución del diagnóstico pre y posnatal del síndrome de Down (SD) durante los periodos $1991-4$ y $2006-9$

\begin{tabular}{|c|c|c|c|c|c|c|c|}
\hline & \multirow{2}{*}{$\begin{array}{l}\text { Periodo de } \\
\text { estudio }\end{array}$} & \multicolumn{3}{|c|}{ Diagnóstico PREnatal } & \multicolumn{3}{|c|}{ Diagnóstico POSTnatal } \\
\hline & & $<35$ años & $\geq 35$ años & Total & $<35$ años & $\geq 35$ años & Total \\
\hline \multirow{2}{*}{$\begin{array}{l}\text { Casos de SD } \\
\text { diagnosticados }\end{array}$} & $1991-4$ & $\begin{array}{c}1 \\
(17 \%)\end{array}$ & $\begin{array}{c}5 \\
(83 \%)\end{array}$ & $\begin{array}{c}6 \\
(100 \%)\end{array}$ & $\begin{array}{c}21 \\
(66 \%)\end{array}$ & $\begin{array}{c}11 \\
(34 \%)\end{array}$ & $\begin{array}{c}33 \\
(100 \%)\end{array}$ \\
\hline & $2006-9$ & $\begin{array}{c}20 \\
(28 \%)\end{array}$ & $\begin{array}{c}52 \\
(72 \%)\end{array}$ & $\begin{array}{c}72 \\
(100 \%)\end{array}$ & $\begin{array}{c}18 \\
(45 \%)\end{array}$ & $\begin{array}{c}22 \\
(55 \%)\end{array}$ & $\begin{array}{c}40 \\
(100 \%)\end{array}$ \\
\hline \multirow{2}{*}{$\begin{array}{l}\text { Número de } \\
\mathrm{SD} / 10.000 \\
\text { partos }\end{array}$} & $1991-4$ & $\begin{array}{c}1 / 16063 \\
0,6\end{array}$ & $\begin{array}{c}6 / 2613 \\
23,0\end{array}$ & & $\begin{array}{c}21 / 16063 \\
13,1\end{array}$ & $\begin{array}{c}11 / 2613 \\
42,1\end{array}$ & \\
\hline & $2006-9$ & $\begin{array}{c}20 / 16951 \\
11,8\end{array}$ & $\begin{array}{c}52 / 10067 \\
51,7\end{array}$ & & $\begin{array}{c}18 / 16951 \\
10,6\end{array}$ & $\begin{array}{c}22 / 10067 \\
21,8\end{array}$ & \\
\hline \multirow{2}{*}{$\begin{array}{l}\text { Edad media } \\
\text { materna (años) }\end{array}$} & $1991-4$ & & $38,86 \pm 3,04$ & & & $32,75 \pm 5,02$ & \\
\hline & $2006-9$ & & $36,08 \pm 4,47$ & & & $34,81 \pm 4,82$ & \\
\hline
\end{tabular}

Durante los 19 años de estudio se diagnosticaron un total de 317 casos con SD, un $55 \%$ de ellos durante el periodo antenatal (Tabla 2). La tasa de detección prenatal del SD muestra una tendencia ascendente a lo largo del periodo de estudio, siendo de un $15,8 \%(6 / 39)$ sobre el total de casos en el primer periodo y de un $64,3 \%$ (72/112) en el último (Tabla 3). Sin embargo, el número de procedimientos invasivos por caso de SD diagnosticado prenatalmente no varió significativamente a lo largo del periodo de estudio (49:1).

La prevalencia total del SD aumentó significativamente $(\mathrm{p}<0,001)$ a lo largo del perio- do de estudio, siendo de un 17,64 por 10.000 en el primer periodo y de 42,80 por 10.000 en el último (Fig. 4). Sin embargo, su prevalencia al nacimiento muestra un discreto descenso, estadísticamente no significativo $(\mathrm{p}=0,31)$, situándose en un 15,76 por 10.000 en el último periodo del estudio. Estos resultados se asocian a un incremento llamativo de la edad materna, circunstancia que conlleva un mayor número de gestaciones con SD. La figura 1b representa el número de recién nacidos con esta cromosomopatía (cálculo realizado según estimación de Morris y $\mathrm{col}^{10}$ ) que habría habido en Navarra en ausencia de programas de detección prenatal.

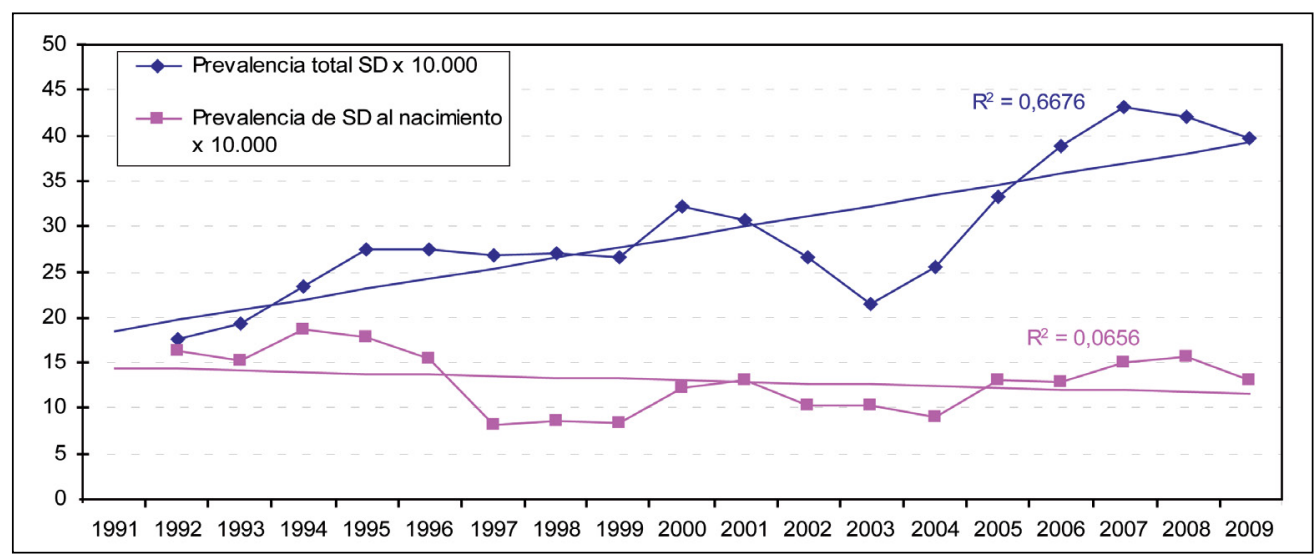

Figura 4. Evolución de la prevalencia total y al nacimiento del síndrome de Down (SD) en Navarra durante el periodo 1991-2009. 


\section{Edad materna de los casos con SD}

Un $17 \%$ de los embarazos con SD diagnosticados prenatalmente entre 1991-1994 correspondieron a madres jóvenes, incrementando hasta un $28 \%$ en el último periodo del estudio. Por el contrario, en el grupo de SD recién nacidos esta proporción disminuyó desde un $66 \%$ hasta un $45 \%$ (Tabla 3) durante el mismo periodo de tiempo. Consecuentemente, la tasa de SD diagnosticados prenatalmente en madres jóvenes en relación al número total de partos fue 20 veces mayor en 2006-9 que en 1991-4; este incremento fue ligeramente superior al doble en el grupo de madres de $\geq 35$ años. La edad media de las madres de niños nacidos con SD aumentó de $32,75 \pm 5,02$ años en el primer periodo (1991-1994) a $34,81 \pm 4,82$ años en el último (2006-2009), en tanto que la de los casos diagnosticados prenatalmente disminuyó de $38,86 \pm 3,04$ a $36,08 \pm 4,47$ años en los mismos periodos.

\section{DISCUSIÓN}

El estudio demuestra claramente el cambio y evolución en Navarra de los dos factores que mayoritariamente influencian la prevalencia al nacimiento del SD, la distribución de edad materna en la población y la tasa de detección de cromosomopatías durante la gestación. El incremento en la edad materna desde 1991 a 2009 hubiera conllevado un aumento del $50 \%$ en el número de recién nacidos con $\mathrm{SD}$, con una prevalencia estimada al nacimiento de un $18,4 / 10.000$ durante la primera parte del periodo del estudio y de un 26,5/10.000 durante la última. El desarrollo de los programas de cribado y diagnóstico prenatal ha contribuido eficientemente a prevenir esta cromosomopatía, si bien su prevalencia al nacimiento no varía significativamente a lo largo de los 19 años, siendo de un 15,76 por 10.000 en el último periodo del estudio. Estos resultados en nuestra comunidad son similares a los observados en otras poblaciones consideradas pioneras en la implementación y desarrollo de programas de prevención de defectos congénitos, tales como Inglaterra y Gales ${ }^{11}$, Estados Uni- $\operatorname{dos}^{12,13}$, Francia $^{14}$, Bélgica ${ }^{15}$ o Países Bajos ${ }^{16}$. La comparación de nuestros resultados con los de otras comunidades autónomas del Estado Español no resulta fácil, dada la escasez de estudios poblacionales y las diferencias autonómicas en la definición y desarrollo de programas de cribado y diagnóstico prenatal de cromosomopatías. La Comunidad de Asturias ${ }^{17}$ describe cambios similares en la prevalencia de SD durante los años 1990-2004, si bien las cifras, tanto de prevalencia total como al nacimiento, son inferiores a las observadas en Navarra. Por otro lado, el ECEMC ${ }^{18}$ describe un descenso significativo de la frecuencia del SD en diversas comunidades autónomas entre los periodos 1980-1985 y 1986-2006, con una incidencia global en este último periodo de un 10,50 por 10.000 y una importante variabilidad interregional. Es muy probable que estas diferencias sean secundarias a las características de ese registro (base hospitalaria) y a su menor (22\%) y desigual cobertura (entre un 6\% y un $78 \%$ ) en las distintas comunidades autónomas.

Como era previsible, el programa de prevención del SD en Navarra ha condicionado un cambio importante en las características socio demográficas asociadas a dicha cromosomopatía. Por un lado, el porcentaje de diagnósticos prenatales de SD se incrementó significativamente en ambos grupos de edad materna a lo largo del periodo de estudio, especialmente entre las menores de 35 años. Así, en este grupo, el número de SD por 10.000 nacimientos diagnosticados prenatalmente fue veinte veces mayor en el último periodo de estudio que en el primero, en tanto que solo se duplicó en el grupo de madres de edad avanzada (igual o mayor de 35). Estos cambios contribuyeron a aumentar la edad materna de los recién nacidos con SD y a disminuir su prevalencia al nacimiento en aproximadamente un $20 \%$ en el grupo de madres jóvenes y en un $50 \%$ entre las de $\geq 35$ años (Tabla 2). Hay que tener en cuenta, sin embargo, que este efecto del programa de diagnóstico prenatal está sobrestimado ya que un $25 \%$ aproximadamente de las gestaciones con SD diagnosticadas por amniocentesis finalizarían espontáneamente de forma 
prematura $^{1}$. En cualquier caso, los cambios secundarios al desarrollo del programa de cribado de SD y su rápida aceptación entre la población gestante de Navarra, son semejantes a los observados en otras poblaciones y constituye un factor fundamental en la prevención de esta cromosomopatía.

Un aspecto de gran interés en este estudio es su capacidad para determinar la cobertura poblacional del programa de cribado y diagnóstico del SD y el grado de aceptación del mismo por parte de las gestantes. El número de gestantes que realizaron un estudio de cribado y/o diagnóstico de cromosomopatías incrementó paulatinamente a lo largo del periodo de estudio, si bien los datos sugieren una tendencia a mantenerse en torno al 70\%. Este porcentaje es superior a las predicciones estimadas por Nicholson y Alberman en $1992^{19}$ y las descritas posteriormente en otras poblaciones ${ }^{15,20,21}$. Es probable que nuestros resultados sean consecuencia de una combinación de factores, tales como un adecuado control de la población gestante, una mayor cobertura de los servicios de Consejo Genético, así como el fácil acceso y la completa gratuidad de los servicios, circunstancias que no siempre confluyen en otras poblaciones. La comparación de nuestros resultados con los de otras comunidades autónomas resulta difícil, dado el escaso número de estudios poblacionales de cobertura publicados al respecto.

Los datos de cobertura del programa de cribado y diagnóstico prenatal del SD en relación al total de partos en Navarra son concordantes con los resultados de la decisión tomada por más de 21.000 gestantes controladas consecutivamente a través de los centros de Atención a la Mujer del área de Pamplona entre los años 2006 y 2009. Es interesante señalar que un $18 \%$ de las gestantes decidieron no participar en el cribado bioquímico o diagnóstico prenatal de cromosomopatías mediante amniocentesis, si bien aceptaban el cribado ecográfico de primer y segundo trimestre; este porcentaje de rechazos fue ligeramente superior entre las madres mayores de 38 años, circunstancia contraria a la descrita en otras poblaciones ${ }^{22}$. Por otro lado, el porcentaje de mujeres que expresó su deseo, en el primer trimestre de gestación, de realizar un test de cribado de segundo trimestre es un $10 \%$ inferior al que realmente lo realizó. Esta diferencia, aunque parcialmente atribuible al riesgo de aborto espontáneo, sugiere que un porcentaje importante de mujeres cambia de opinión o de circunstancias personales en el transcurso de las tres o cuatro semanas que media entre la obtención de la información y su definitiva decisión de realizar el cribado de segundo trimestre. Estos datos reflejan y apoyan la relevancia de realizar un adecuado proceso de información que facilite la toma de decisiones de forma autónoma por parte de las mujeres gestantes.

Las $2 / 3$ partes (28/42) de los niños nacidos con SD correspondieron a embarazos cuyas madres no quisieron realizar un test de cribado o una amniocentesis; de ellos, un $9,5 \%(n=4)$ presentaban signos ecográficos altamente sugestivos de cromosomopatías y otros 7 (17\%) tuvieron un resultado positivo en el test de cribado de SD. De estos resultados se deduce que en nuestra población y durante el último periodo de estudio, una cuarta parte del total de niños nacidos con SD se diagnosticaron prenatalmente o tuvieron un test de cribado bioquímico/ecográfico positivo, si bien las madres decidieron continuar con el embarazo; un $16,5 \%$ adicional fueron falsos negativos del test de cribado. Estos datos son de gran importancia a la hora de planificar futuros programas de prevención y cuidado postnatal de los niños con SD, ya que el cambio social en la percepción y aceptación de la discapacidad asociada a esta cromosomopatía puede ser un factor relevante a tener en cuenta en el futuro.

Uno de los puntos fuertes de este estudio es su carácter poblacional y la alta calidad de los datos referentes a las gestaciones y su producto final, dada la coordinación centralizada de los programas de cribado, diagnóstico prenatal y registro de defectos congénitos de base poblacional. La capacidad de detección de los casos con SD es prácticamente total ya que todas las gestaciones, cariotipos fetales 
(pre y postnatales) y nacimientos del sistema público de salud (más del $90 \%$ del total) se controlan, desde 1991, a través de las fuetes de información utilizadas para la realización de este estudio. Para la identificación del resto de los casos se han consultado el CMBD (Conjunto Mínimo Básico de Datos), la historia Clínica Informatizada del CHN y la Asociación de SD de Navarra. Estas circunstancias, junto a una correcta estandarización y universalización de los programas de prevención de cromosomopatías, son los elementos esenciales para evaluar su impacto y aceptación en la población y definir, en último lugar, planes de prevención (primaria y secundaria) y cuidado postnatal, basados en las necesidades de la población y la adecuada relación coste-beneficio,

\section{Agradecimientos}

A todos los colaboradores del programa de cribado y diagnóstico prenatal de Navarra, y muy especialmente, al Dr. Ander Arrazola, por su inestimable labor en el proceso de reconocimiento del RACEHNA como Registro Autonómico del Sistema Navarro de Salud. A la Dra. Begoña Bermejo por su ayuda y asesoramiento en el tratamiento estadístico de los datos.

\section{BIBLIOGRAFÍA}

1. Savva GM, Morris JK, Mutton DE, Alberman E. Maternal age-specific fetal loss rates in Down syndrome pregnancies. Prenat Diagn 2006; 26: 499-504.

2. Cuckle HS, Holding S, Jones R, Groome NP, WALLACE EM. Combining inhibin A with existing second-trimester markers in maternal serum screening for Down's syndrome. Prenat Diagn 1996; 16: 1095-1100.

3. Benacerraf BR, Neuberg D, Bromley B, FrigoLETTO FD JR. Sonographic scoring index for prenatal detection of chromosomal abnormalities. J Ultrasound Med 1992; 11: 449-458.

4. Ball RH, Caughey AB, Malone FD, Nyberg DA, Comstock CH, SAADE GR et al. First and Second Trimester Evaluation of Risk (FASTER) Research Consortium. First- and second-trimester evaluation of risk for Down syndrome. Obstet Gynecol 2007; 110: 10-17.
5. Dolk H, Loane M, Garne E, De Walle H, QueisserLuft A, DE Vigan C et al. Trends and geographic inequalities in the prevalence of Down syndrome in Europe, 1980-1999. Rev Epidemiol Sante Publique 2005; 53, 2: 2S87-95.

6. Cocchi G, Gualdi S, Bower C, Halliday J, JonsSON B, MYrELID A et al. International trends of Down syndrome 1993-2004: Births in relation to maternal age and terminations of pregnancies. Birth Defects Res A Clin Mol Teratol 2010; 88: 474-479.

7. Muggi EE, McCloskey D, Halliday JL. Health behaviour modelling for prenatal diagnosis in Australia: a geodemographic framework for health service utilisation and policy development. BMC Health Serv Res 2006; 6: 109.

8. Descripción del estado de situación del cribado prenatal de las cromosmopatías más frecuentes -principalmente del síndrome de Down- en el Estado Español y propuestas de mejora en la práctica clínica habitual. Informes de Evaluación de Tecnologías Sanitarias AATRM Num. 2006/03.

9. Orden Foral 56/2006. B.O. de Navarra de 29 de mayo (publicado el 21 de Julio de 2006).

10. Morris JK, Mutton DE, Alberman E. Revised estimates of the maternal age specific live birth prevalence of Down's syndrome. J Med Screen 2002; 9: 2-6.

11. Morris JK, Alberman E. Trends in Down's syndrome live births and antenatal diagnoses in England and Wales from 1989 to 2008: analysis of data from the National Down Syndrome Cytogenetic Register. BMJ 2009; 339: b3794.

12. Egan JF, Benn PA, Zelop CM, Bolnick A, GianFERRARI E, BORGIDA AF. Down syndrome births in the United States from 1989 to 2001. Am J Obstet Gynecol 2004; 191: 1044-1048.

13. Resta RG. Changing demographics of advanced maternal age (AMA) and the impact on the predicted incidence of Down syndrome in the United States: Implications for prenatal screening and genetic counseling. Am J Med Genet A 2005; 133A: 31-36.

14. Khoshnood B, De Vigan C, Vodovar V, Goujard J, GofFinet F. A population-based evaluation of the impact of antenatal screening for Down's syndrome in France, 1981-2000. Bjog 2004; 111: 485-490.

15. Verloes A, Gillerot Y, Van Maldergem L, Schoos R, Herens C, JAMAR M et al. Major decrease in the incidence of trisomy 21 at birth in south Belgium: mass impact of triple test? Eur J Hum Genet 2001; 9: 1-4. 
16. Weijerman ME, van Furth AM, Vonk NoORdegraAF A, van Wouwe JP, Broers CJ, Gemke RJ. Prevalence, neonatal characteristics, and firstyear mortality of Down syndrome: a national study. J Pediatr 2008; 152: 15-19.

17. Mosquera Tenreiro C, Ariza Hevia F, Rodriguez Dehli C, Fernandez Toral J, García López E, RiaNo GaLAN I. Frecuencia del síndrome de Down en Asturias y tendencia temporal, 1990-2004. Med Clin (Barc) 2009; 132: 580-584.

18. Bermejo E, Cuevas L, Mendioroz J, Grupo PeriféRICO DEL ECEMC, MARTíNEZ-Frías ML. Frecuencia de anomalías congénitas en España: Vigilancia epidemiológica en el ECEMC en el período 1980-2007. Boletín del ECEMC: Revista de Dismorfología y Epidemiología, serie V, $\mathrm{n}^{\mathrm{0}} 7$, 2008: 59-88.
19. Nicholson A, Alberman E. Prediction of the number of Down's syndrome infants to be born in England and Wales up to the year 2000 and their likely survival rates. J Intellect Disabil Res 1992; 36 (Pt 6): 505-517.

20. Gidiri M, McFarlane J, Holding S, Lindow S. Maternal serum screening for Down syndrome: are women's perceptions changing? Bjog 2007; 114: 458-461.

21. Dixon J, Pillai M, Mahendran D, Brooks M. An assessment of the Down syndrome antenatal screening policies of East and West Gloucestershire between 1993 and 1999. J Obstet Gynaecol 2004; 24: 760-764.

22. GiRidi M, Holding S, Lindow SW. Reduction in Down syndrome screening acceptance is predominantly observed in women aged 2535 years. Women's Health 2010, 6: 525-529. 